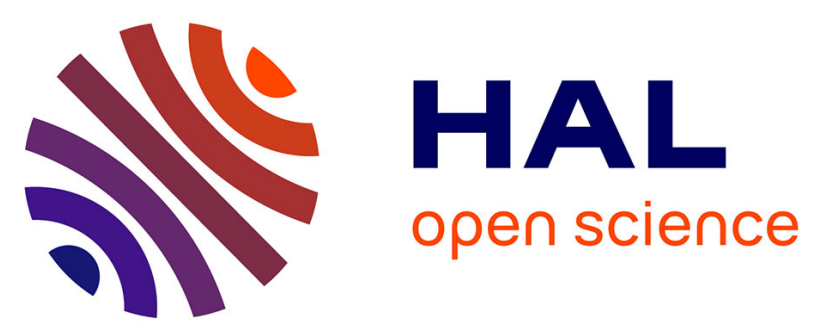

\title{
Diffusion des gobelets bruns d'Argonne entre la fin du Ier siècle et la fin du IIe siècle en Normandie
} Patrick Blaszkiewicz, Daniel Dufournier

\section{To cite this version:}

Patrick Blaszkiewicz, Daniel Dufournier. Diffusion des gobelets bruns d'Argonne entre la fin du Ier siècle et la fin du IIe siècle en Normandie. Gallia - Fouilles et monuments archéologiques en France métropolitaine, 1989, 46, pp.253-259. 10.3406/galia.1989.2898 . hal-01939653

\section{HAL Id: hal-01939653 \\ https://hal.science/hal-01939653}

Submitted on 12 Feb 2020

HAL is a multi-disciplinary open access archive for the deposit and dissemination of scientific research documents, whether they are published or not. The documents may come from teaching and research institutions in France or abroad, or from public or private research centers.
L'archive ouverte pluridisciplinaire HAL, est destinée au dépôt et à la diffusion de documents scientifiques de niveau recherche, publiés ou non, émanant des établissements d'enseignement et de recherche français ou étrangers, des laboratoires publics ou privés.

\section{(ㅇ)(1) $\$$}

Distributed under a Creative Commons Attribution - NonCommercial - NoDerivatives| 4.0 


\title{
DIFFUSION DES GOBELETS BRUNS D'ARGONNE ENTRE LA FIN DU I' ${ }^{\text {er }}$ SIÈCLE ET LA FIN DU II SIÈCLE EN NORMANDIE
}

\author{
par Patrick BLASZKIEWICZ et Daniel DUFOURNIER
}

Peu de chercheurs s'étaient véritablement intéressés depuis $\mathrm{G}$. Chenet aux problèmes que soulèvent les gobelels à paroi fine produits en Gaule du Nord.

Un essai de synthèse a été publié au début des années 1980 par A. C. Anderson.

Le recensement des gobelets, leur caractérisation physico-chimique nous a conduit à reconsidérer certaines dés propositions avancées par A. C. Anderson pour ce qui concerne les origines de fabrication, leurs datations et loms diffusions.

Les analyses ont porté sur dix sites fouillés récemment.

Sur une soixantaine de gobelets analysés, cinquante sont apparentés aux productions d'Argonne, le reste ér.:- constitué par les ateliers Mosellans et le Centre de la France.

(\%) Parmi ces 50 gobelets, une dizaine de pièces avait des traces vrillées à l'intérieur au niveau du col (ces traces vrillées selon A. C. Anderson caractérisaient l'atelier de Compiègne). Il est donc évident que ces gobelets dits de Compiègne, ne peuvent provenir que de la région d'Argonne, donc qu'il n'existe pas d'atelier à Compiègne ayant produit ce type de céramique.

La plupart de ces gobelets, qu'ils soient d'Argonne, de Sinzig ou de Colchester ont une morphologie tout à fait comparable, à savoir une couleur tirant sur le brun, un dépôt de granules d'argile, et des lèvres plus ou moins saillantes «en corniche». Ce qui prouve s'il en était besoin que les seuls critères de forme et de décor pour des productions aussi spécifiques sont tout à fait aléatoires pour distinguer des origines de fabrication.

Après comparaison avec le matériel associé, il semblerait qu'il faille élargir la fourchette chronologique, donc que ces gobelets auraient été exportés au moins depuis la fin du $I^{\text {er }} \mathrm{s}$. jusqu'à la fin du $\mathrm{II}^{\mathrm{e}} \mathrm{s}$.

Quant à la diffusion, il est évident que ces gobelets sont beaucoup plus nombreux en Haute-Normandie tant numériquement que proportionnellement, phénomène qui se retrouve parfaitement dans la diffusion d'Argonne moulé, donc qu'ils participeraient à un même courant commercial que la sigillée moulée et que les céramiques Gallo-Belge du ier $\mathrm{s}$.

Few scientist had really been interested, since G. Chenet in the problems raised by fine ware beakers made in Northern Gaul.

A synthetical attempt was published in the early $80 \mathrm{~s}$ by A. C. Anderson. The inventory of these goblets, their chemical characterization have lead us to reconsider some suggestions that were put forward by A. C. Anderson, that is to say the origins of their making, their dates and their distribution.

In order to do this we have analysed about sixty of these beakers, fifty of which have been identified as coming from Argonne, the others being composed of heterogenous productions (Moselle, Bourgogne, Centre).

Among the fifty goblets from Argonne, ten of them had twisted marks on the inside of the neck-lip junction, these fine ware beakers were typical, according to A. C. Anderson of the products of Compiegne. Therefore the result of the chemical analyses proves that the clay which was used was the same for the beakers from Argonne and for the assumed workshop of Compiène. That it is almost certain that no workshop of Compiègne could produce this type of pottery. 
Most of these beakers, whether from Argonne, Sinzig or from Colchester have a completely similar morphology which proves that the only morphological crileria for so specific productions are enlirely uncertain to distinguish the origins of the making.

After comparing with the associated material (smooth Samian ware from Argonne) it seems that one should extend the chronological scale which means that these goblets should have been exported at least from the end of the Ist century to the end of the IInd century.

As to the distribution of these goblets, it is obvious that these potteries are much more numerous in Upper Normandy, a phenomenon which occurs for the moulded Samian ware from Argonne and the Gallo-Belgic potteries of the Ist century and which would lake part in the same East Western commercial movement.

Depuis les fouilles d'A. de Roucy en forêt de Compiègne dans les années $1860^{1}$ et les découvertes très importantes de G. Chenet au début du xx $x^{\mathrm{c}}$ s. en forêt d'Argonne ${ }^{2}$ - ateliers de Lavoye, Les Allieux et Avocourt - peu de chercheurs se sont véritablement intéressés aux problèmes que soulève l'étude des gobelets à paroi mince produits en Gaule du Nord : qu'il s'agisse des origines de fabrication, de la datation ou de la diffusion.

Il a effectivement fallu attendre les années 1980, avec les travaux de A. C. Anderson ${ }^{3}$ pour voir publier un essai de synthèse concernant les centres de production de gobelets à paroi fine dans les provinces du Nord-Ouest de l'Empire. L'auteur distingue dix-neuf centres de production dont deux au moins seraient situés en Gaule du Nord : Compiègne et/ou Jaulges-Villiers-Vineux d'une part, la forêt d'Argonne d'autre part.

Le recensement des gobelets recueillis en Normandie ${ }^{4}$, leur caractérisation physico-chimique et les

1 Ces fouilles n'ont, semble-t-il, concerné aucun atelier de fabrication de gobelets.

2 Ces découvertes ont fait l'objet de plusieurs publications. Pour les gobelets citons notamment G. Chenfe, Gobelets ovoïdes moulés d'Autry-Lavoye, Pro Alesia, V, 1920, p. 129-141. Une grande partie de ces gobelets est déposée au Musée des Antiquités nationales de Saint-Germain-en-Laye. Pour une synthèse des fouilles et découvertes de G. Chenet en Argonne, cf. G. Chenet et G. Gaudron, La céramique sigillée d'Argonne des $I^{\prime}$ et $H^{\mathrm{e}}$ siècles, $6^{\mathrm{e}}$ suppl. à Gallia, 1955, $249 \mathrm{p}$.

3 A. C. Anderson, A guide to Roman Fine Ware, Highworth, Wiltshire, 1980. Il existe une traduction française de cet ouvrage : J. M. Perrin et R. Sénéchal, Guide de la céramique romaine à parois fines, Revue archéologique Siles, Hors série no 17, 1985, $114 \mathrm{p}$. Voir encore A. C. Anderson, Some continental beakers of the First and Second centuries AD, in : A. C. et A. S. Anderson, Roman Potlery Research in Britain and North-West Europe, papers presented to Graham Webster, BAR Internalional Series 123 (ii), 1981, pp. 321-347.

4 Plus de 400 échantillons de gobelets découverts en Haute et Basse-Normandie ont été rassemblés pour une étude premiers éléments d'une enquête sur le contexte de leur découverte nous conduisent à reconsidérer certaines des propositions avancées par A. C. Anderson tant en ce qui concerne les origines de fabrication que la datation et la diffusion des gobelets ovoïdes bruns fabriqués en Gaule du Nord.

Vingt-quatre sites normands ayant fourni ce type de gobelets ont été recensés (fig. 1): douze nécropoles, quatre sites d'habitat groupé, trois villae, un fanum et quatre indéterminés. Neuf d'entre eux ont été fouillés récemment, les autres l'ont été il y a près d'un siècle. Les gobelets recueillis sur ces différents sites n'ont pas tous fait l'objet d'une étude détaillée mais presque tous ont été examinés. Les analyses concernent dix sites; elles ont notamment porté sur le matériel découvert au cours des fouilles les plus récentes.

\section{CARACTÉRISATION CHIMIQUE DES GOBELETS BRUNS RECUEILLIS EN NORMANDIE, ORIGINES DE FABRICATION}

Les 61 gobelets analysés se répartissent en quatre groupes de composition chimique parfaitement distincts (cf. tableau, p. 259). Leur répartition apparait cependant très inégale : le premier groupe réunit 50 gobelets alors que les trois autres n'en rassemblent respectivement que 6,3 et 2 . L'échantil-

générale. Sur cet ensemble plus de la moitié était constituée de gobelets à surface grise et pâte blanche. Ces dernières pièces ont très probablement été fabriquées en Normandie et n'ont pas été prises en compte dans cet article. Voir à leur propos : P. Bi.aszikiewicz et D). Dufournifr, Caractérisation et diffusion du "gobelet sac" en Normandie entre la fin du ret la fin du ue siècle, à paraître dans les Actes du congrès de la Société française d'Étude de la Céramique antique en Gaule (SFECAG), tenu à Caen en 1987. 
Fig. 1 - Carte de répartition des gobelets et des sigillées moulées estampillées d'Argonne découverts en Normandie. L'aire des carrés est proportionnelle au nombre de gobelets découverts (pour Rouen, 200 gobelets environ).

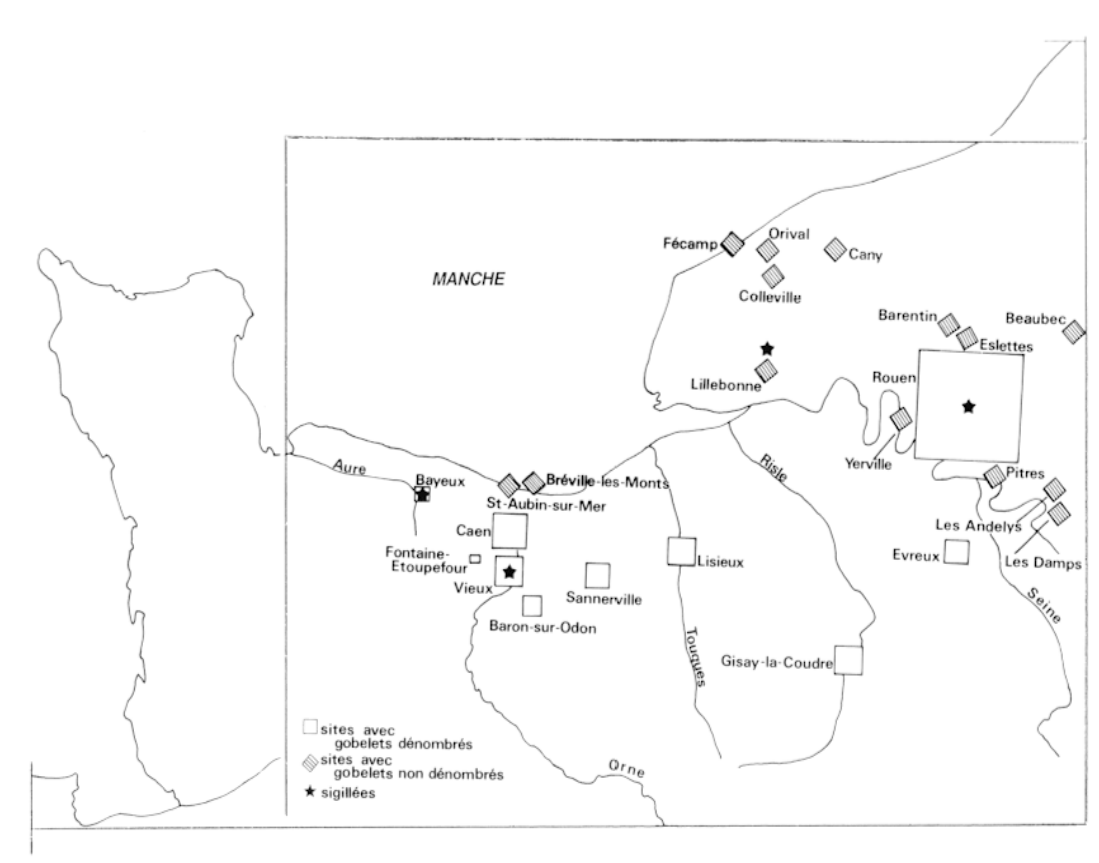

lonnage opéré avant l'analyse n'était pas parfaitement cohérent, toutefois il ne fait guère de doute que la disproportion numérique observée entre le premier groupe et les suivants reflète, à peu de chose près, la réalité.

La comparaison des résultats dianalyse avec tous ceux dont nous avons pu disposer par ailleurs ${ }^{5}$ a permis d'apparenter les 50 échantillons du premier groupe aux productions d'Argonne, les 6 échantillons du second aux ateliers mosellans, les 3 suivants aux productions de Jaulges-Villiers-Vineux et les 2 derniers à celles du Centre, vraisemblablement Lezoux ${ }^{6}$.

La très grande majorité des gobelets bruns recueillis en Normandie provient donc des ateliers

5 F. A. Hart, J. M. V. Storey, S. J. Adams, R. P. SYMonds, J. M. WALSh, An analytical study, using inductively coupled plasma (ICP) spectrometry of Samian and colour-coated wares from the Roman Town at Colchester together with related Continental Samian wares, Journal of Archaeological Science, 14, 1987, à paraitre; - M. Picon, Introduction à l'étude technique des céramiques sigillées de Lezoux, Centre de Recherches sur les Techniques grécoromaines, université de Dijon, 2, 1973, 135 p.; A. M. Pollard, H. Hatchfr, R. P. Symonds, Provenance studies of Rhenish Pottery by comparison with terra sigillata, Revue d'Archéométrie, 5, 1981, p. 177-185;-D. Dufournita, C. Pilfer, La céramique de Frénouville, nécropole des IveVII siècles : premiers résultats des analyses typologiques et chimiques, Actes du 105' Congrès national des Sociétés savantes (Caen, 1980), Paris, 1983, p. 85-98.

6 Pour les gobelets ne provenant pas d'Argonne. Voir P. Biaszikifwicz, et D. Difournifr, op. cit., note 4.
d'Argonne. Cette observation en appelle immédiatement une seconde : si un atelier compiègnois avait également produit des gobelets à paroi fine il serait surprenant de n'en trouver aucune trace en Normandie et plus particulièrement à Rouen. Or nos résultats montrent que tous les gobelets bruns analysés ont trouvé leur origine de fabrication c'està-dire qu'aucun d'entre eux n'a présenté une composition chimique marginale que l'on aurait alors pu apparenter à un atelier différent des précédents et peut-être encore inconnu.

A. C. Anderson relève une ressemblance morphologique frappante entre les productions d'Argonne et les productions présumées de Compiègne, distinguant celles-ci des premières grâce, notamment, à la présence de traces en vrille situées à l'intérieur du gobelet, au niveau du col (fig. 2)? Comme une partie des pièces analysées appartenant au premier groupe présente de telles traces, il faudrait alors supposer que ces deux ateliers ont utilisé une matière première argileuse identique, à la fois présente en Argonne et en forêt de Compiègne. Une telle hypothèse ne tient pas lorsque l'on considère de près les résultats d'analyse : outre la très forte homogénéité des résultats - illustrée par la faible valeur des écarts-types [E-T] — nous n'avons en effet relevé aucune différence

7 De telles traces ne présentent en réalité aucune originalité. Elles sont en effet très courantes sur les poteries tournées et relèvent de l'opération de tournage elle-mème. 


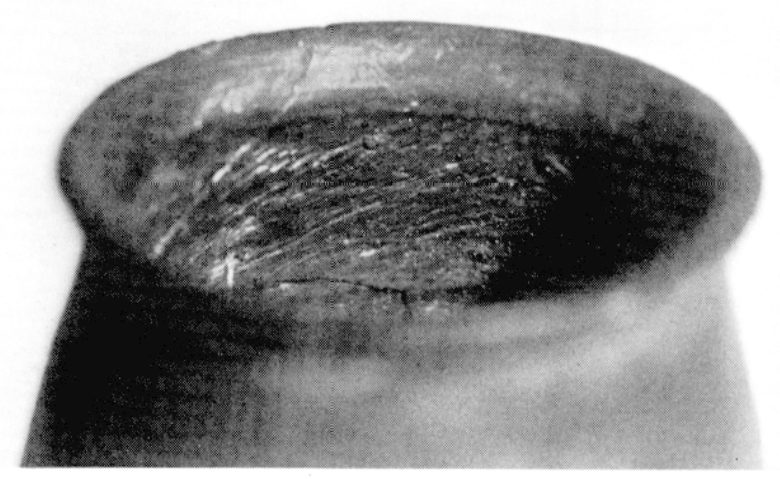

Fig. 2. - Traces vrillées visibles à l'intérieur de certains gobelets.

statistiquement significative entre les gobelets vrillés et les autres.

Il est donc à peu près établi qu'aucun atelier situé en forêt de Compiègne n'a produit de gobelets bruns à paroi fine et que seuls, en Gaule du Nord, les ateliers d'Argonne ont massivement exporté leurs produits vers l'Ouest.

\section{DESCRIPTION MORPHOLOGIQUE SUCCINCTE DES GOBELETS D'ARGONNE}

La plupart des gobelets d'Argonne ${ }^{8}$ ont en commun une surface externe brun foncé rendue très rugueuse par le dépôt volontaire, à cru, de granules d'argile ${ }^{9}$. Cet aspect caractérise d'ailleurs les productions de nombreux autres ateliers. La concentration de ces granules est extrêmement variable d'un gobelet à l'autre. La partie comprise entre cette zone rugueuse et la lèvre montre toujours des traces de polissage. Ces deux zones sont parfois délimitées par une rainure.

Ces gobelets se présentent sous trois formes ${ }^{10}$ :

- la forme dite "tassée», (exemple fig. $\left.3, \mathrm{n}^{\circ} 15\right)$;

- la forme dite "élancée», (exemple fig. 3, $\left.n^{\circ} 5\right)$

8 Pour de plus amples informations nous renvoyons le lecteur aux articles et ouvrages déjà cités en notes 2,3 et 4 . Les dessins nos 10 à 15 de la fig. 3 nous ont été aimablement communiqués par R. P. Symonds et S. Wade. Nous les en remercions très vivement.

9 A. C. Anderson (op. cil., note 3) propose un certain nombre de possibilités techniques qu'il ne nous a pas semblé indispensable de rappeler et de discuter ici. Notons que cet aspect granuleux se traduit selon les auteurs par des termes variés : "gobelet sablé", "gobelet granité"...

10 Nous reprenons ici la terminologie proposée par A. C. Anderson (op. cit., note 3). $\left.n^{\circ} 1\right)$.

- la forme dite «à dépression», (exemple fig. 3,

Les lèvres plus ou moins saillantes sont dites en "corniche", une rainure plus ou moins profonde et nette en marque la face externe. L'ètat fragmentaire du mobilier n'a pas permis de distinguer différents types de fonds.

Ces données morphologiques se révèlent en pratique assez peu spécifiques; elles ne permettent pas par exemple de distinguer avec certitude les gobelets fabriqués en Argonne des productions de Sinzig en Rhénanie ou de Colchester en Angleterre ${ }^{11}$ (fig. 3, $n^{\circ} 6$ ). Cela prouve s'il en était encore besoin que les seuls critères de forme, de couleur et de décor sont très souvent insuffisants pour distinguer les origines de fabrication.

\section{CONTEXTE DES DÉCOUVERTES NORMANDES ESSAI DE DATATION}

Pour contribuer à une meilleure datation de la production des gobelets d'Argonne nous nous sommes référés au matériel qui leur était associé sur trois des sites les plus récemment fouillés, qui représentent trois types d'occupation, ainsi qu'à celui découvert dans la nécropole gallo-romaine du GrandJardin à Lisieux (Calvados) fouillée au $\mathrm{XIX}^{\mathrm{e}}$ s., dont le mobilier céramique a été publić en $1986^{12}$. Les trois premiers sites concernent une villa à FontaineÉtoupefour (Calvados) ${ }^{13}$, un vicus de tanneurs à Caen (Calvados) ${ }^{\mathbf{1 4}}$ et un habitat urbain à Rouen (SeineMaritime $)^{15}$. Une attention particulière a été portée aux sigillées moulées et estampillées d'Argonne qui

11 Voir à ce propos : R. P. Symonds, Le problème des vases ovoïdes, à paraître dans les Actes du Congrès de la SFECAG, tenu à Caen en 1987.

12 P. Blastikiewicz, P. David, C. Jigan, J.-Y. Marin, Quelques données nouvelles sur la nécropole gallo-romaine du Grand-Jardin à Lisieux (Calvados) : La collection Delaporte du Musée de Lille, Revue archéologique de l'Ouest, 3, 1986, p. 119 134.

13 Fouilles inédites de D. Bertin en 1976.

14 Fouilles de J.-Y. Marin de 1979 à 1981, cf. C. JIGAN, J.-Y. Marin, Archéologie urbaine à Caen, Publications du Musée de Normandie, 2, Caen, 1981.

15 Fouilles de P. Halbout de 1978 à 1981, cf. P. HaLbout et alii, Rouen gallo-romain: fouilles et recherches archéologiques, Catalogue de l'exposition de l'association Rouen Archéologie, Musée des Beaux-Arts de Rouen, 24 septembre-30 novembre 1982, Rouen, Ed. Association Rouen archéologie, 1982. 

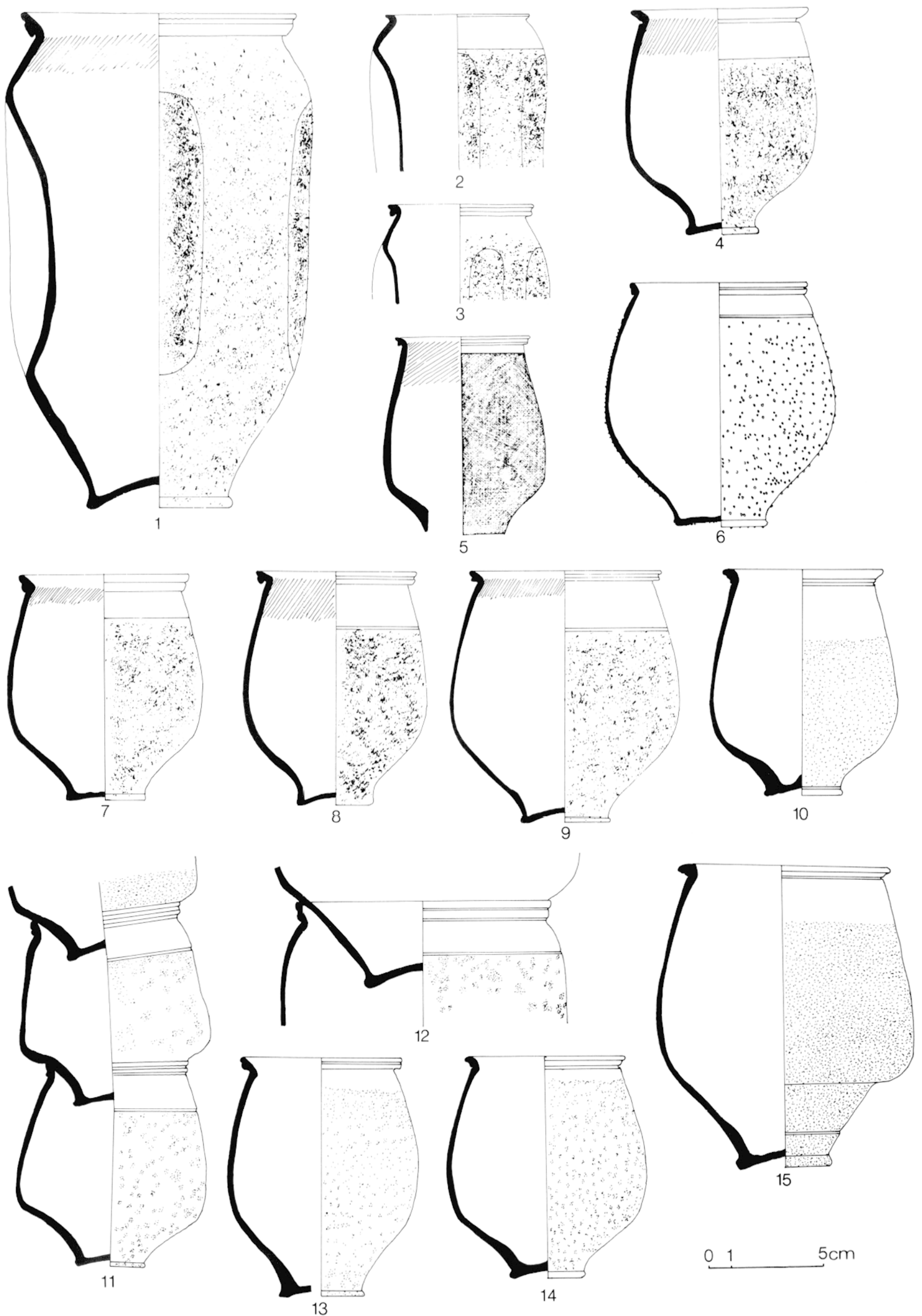

Fig. 3 - Gobelets produits en Argonne (nos 1-5: Rouen; 7-9: Lisieux; 10-15: Lavoye ; 11-12: Avocourt; 13-14: Argonne) et à Colchester ( $n^{\circ} 6$ production). Les gobelets $n^{\circ} 1,5$ et 8 ont été analysés; les nos $1-3$ sont à dépression; les nos $10-15$ proviennent d'ateliers situés en forêt d'Argonne (collection Chenet au Musée des Antiquités nationales de Saint-Germain-en-Laye). 
ont très probablement èté produites et exportées en même temps que les gobelets ${ }^{16}$.

Fontaine-Étoupefour (Calvados) : le matériel associé découvert dans cette villa est principalement composé de sigillées importées du Centre de la Gaule et que D. Bertin a daté du $I^{e} s$. Aucune sigillée moulée d'Argonne n'y a été découverte.

Caen (Calvados), vicus de tanneurs : les gobelets ont été recueillis dans un dépotoir avec dix sigillées provenant du Centre de la Gaule. Les estampilles, notamment DOECCUS et CASURIUS, qui les caractérisent appartiennent à la seconde moitié du $\mathrm{II}^{\mathrm{e}} \mathrm{s}^{17}$. La fouille n'a mis au jour aucune sigillée d'Argonne.

Rouen (Seine-Maritime) : les nombreux gobelets d'Argonne découverts à Rouen sont associés à un mobilier céramique composé de sigillées moulées et estampillées également produites en Argonne ainsi que de sigillées du Centre. Une quinzaine d'estampilles a été relevée sur les premières parmi lesquelles : TRIBUNUS (140-180), TOCCA (120-160), TULLUS (120-160) et CRACISA (120-160). Elles correspondent toutes à ce que l'on sait des productions d'Argonne du $\mathbf{I I}^{\mathrm{e}} \mathrm{s}^{18}$. Toutes ces découvertes sont réparties dans des niveaux datés de 80 à $200^{19}$.

Lisieux (Calvados), nécropole du Grand-Jardin (secteur 2): un verre signé F.I.R.M. datable du ${ } \mathbf{I}^{*} \mathbf{s .}{ }^{20}$, deux sigillées à décor excisé du Centre de la Gaule, de la fin du I $^{*}$ s. ou du II $^{\mathrm{e}} \mathrm{s}^{21}$, et une coupelle sigillée estampillée MARTIO, également du Centre, datable entre 140 et $190^{22}$ sont les objets les plus marquants qu'il a été possible d'associer aux gobelets d'Argonne. Là encore, aucune sigillée moulée d'Argonne n'appartient au mobilier recueilli dans cette nécropole.

16 Voir notamment les productions de l'atelier du Vault-Mulard dans G. Chenet et G. Gaudron, op. cit., note 2, ainsi que R. P. Symonds, Rhenish Ware, Five darkcoloured pollery from Gaul and Germany, thèse de Doctorat, université d'Oxford, Oxford Commitee for Archaeology Monograph, 23, à paraitre.

17 J. H. Stanfield, G. Simpson, Central Gaulish Potlers, Londres, 1958.

18 G. Chentet et G. Gaudron, op. cit., note 2 ; B. Hormann, Catalogue des estampilles sur vaisselle sigillee, Notice lechnique, Groupe d'Archéologie antique du Touring Club de France, 22, 1979, 36 p.; - B. Iofmann, Catalogue des poinçons pour moules à vases sigillés des décorateurs argonnais, $O G A M, 20,1968$, p. 273-307.

19 C. JIGAN, J.-Y. MARIN, op. cit., note 14 et communication personnelle de $P$. Halbout.

20 P. Btaszkifvicz et alii, op. cil., note 12, p. 131, pl. IX, no 7 .

21 Ibid., p. 126, pl. IV, nox 3 et 4.

22 Ibid., p. 127
D'après l'ensemble de ces données, les gobelets en Argonne auraient donc été exportés vers la Normandie au moins depuis la fin du $\mathrm{I}^{\text {er }} \mathrm{s}$. jusqu'à la fin $\mathrm{du} \mathbf{I I}^{\mathrm{e}} \mathrm{s}$. Cette période apparaît beaucoup plus large que celle jusqu'à présent proposée par A. C. Anderson pour la production de ces gobelets.

\section{REMARQUES GÉNÉRALES SUR LA DIFFUSION DES GOBELETS D'ARGONNE EN NORMANDIE}

La carte proposée (fig. 1) montre clairement que les gobelets produits en Argonne sont beaucoup plus nombreux en Haute-Normandie, notamment le long du couloir de la Seine, qu'en Basse-Normandie. Précisons en outre que leur proportion par rapport au reste du mobilier céramique est aussi beaucoup plus importante dans la première région que dans la seconde. Nous avons pu constater que le mème phénomène caractérisait les sigillées moulées estampillées d'Argonne : très peu nombreuses sur les sites bas-normands elles sont en revanche très répandues dans la vallée de la Seine.

Cette première observation illustrerait bien le fait que ces deux types céramiques participaient d'un même courant de production et de commercialisation.

Cependant les raisons qui permettent, sinon d'expliquer, du moins de comprendre la faible quantité de pièces argonnaises recueillies en BasseNormandie diffèrent selon qu'il s'agit des gobelets ou des sigillées. Pour les premiers, il suffit de rappeler que de très nombreux gobelets d'un autre type, gris à pâte blanchâtre, sont à la même époque utilisés sur les sites bas-normands et qu'ils sont probablement d'origine locale ${ }^{23}$. En ce qui concerne les sigillées, les productions argonnaises semblent avoir été très largement concurrencées par celles de la Gaule centrale qui prédominent sur les sites bas-normands.

Les travaux de A. C. Anderson ${ }^{24}$ et ceux de R. P. Symonds ${ }^{25}$ montrent que les gobelets et les sigillées estampillées d'Argonne sont aussi peu représentées dans le Sud de l'Angleterre qu'en BasseNormandie.

La quasi-absence de ce mobilier dans tous les dépôts de fouilles visités, situés aussi bien au Sud qu'à l'Ouest de la Basse-Normandie montre que

23 P. Blaszkifwicz, D). DUfournikr, op, cit., note 4.

24 Cif. note 3.

25 R. P. Symonds, op. cit., note 16. 
cette dernière région constitue probablement les limites extrêmes, Sud et Ouest, de la diffusion des céramiques argonnaises.

Il est frappant de constater combien les caractères de cette diffusion sont proches de ceux qui ont marqué, au siècle précédent, l'exportation de la céramique gallo-belge vers la Normandie ${ }^{26}$. Nous pouvons dès lors conjecturer l'existence d'un courant commercial régulier qui, d'Est en Ouest, aurait

26 P. Blaszikifwicz, P. David, Les céramiques gallobelges estampillées du $\mathrm{I}^{\mathrm{er}}$ siècle trouvées en Normandie, à paraître dans les Acles du congrès de la SFECAG, tenu à Caen en 1987. perduré depuis le début du $\mathrm{I}^{\mathrm{er}} \mathrm{s}$. jusqu'au $\mathrm{v}^{\mathrm{e}} \mathrm{s} .{ }^{27}$. Un courant dont la Basse-Normandie aurait souvent constitué l'extrémité ouest ${ }^{28}$ tout en restant ouverte aux importations du Centre.

Patrick Blaszkiewicz et Daniel Dufournier

27 Le III' s. voit l'importation en Haute-Normandie notamment de céramiques fabriquées à Trèves et à Wetterau (Symonds R. P., op. cit., note 16). Pour les ive et ve s., les importations de l'Est sont surtout caractérisées par les sigillées d'Argonne décorées à la molette : P. Halbout, C. Jigan, La céramique d'Argonne décorée à la molette des Iv et ve siècles en Normandie, à paraître dans les Actes du congrès de la SFECAG, tenu à Caen en 1987.

28 Notons pour le Ive $\mathrm{s}$. que la céramique d'Argonne décorée à la molette est bien représentée en Armorique, notamment à Alet, cf. L. Langoü̈r, Les Coriosolites, 1987, p. 239.

Compositions chimiques et origines de fabrication des 61 gobelets bruns analysés.

\begin{tabular}{|l|c|c|c|c|l|l|l|l|l|l|}
\hline Origine & $\mathrm{SiO}_{2}$ & $\mathrm{Al}_{2} \mathrm{O}_{3}$ & $\mathrm{Fe}_{2} \mathrm{O}_{3}$ & $\mathrm{TiO}_{2}$ & $\mathrm{CaO}$ & $\mathrm{MgO}$ & $\mathrm{Na}_{2} \mathrm{O}$ & $\mathrm{K}_{2} \mathrm{O}$ & $\mathrm{MnO}$ & $\mathrm{P}_{2} \mathrm{O}_{5}$ \\
\hline & & & & & & & & & & \\
Argonne $\mathrm{n}=50$ & 66,25 & 19,35 & 6,62 & 1,02 & 1,82 & 1,21 & 0,22 & 3,21 & 0,035 & 0,19 \\
Ecarts-types & 1,22 & 0,45 & 0,38 & 0,03 & 0,90 & 0,09 & 0,04 & 0,17 & 0,008 & 0,13 \\
\hline & & & & & & & & & & \\
Moselle $\mathrm{n}=6$ & 57,95 & 18,53 & 6,75 & 0,83 & 3,75 & 4,60 & 0,23 & 6,98 & 0,066 & 0,32 \\
Ecarts-types & 2,87 & 0,78 & 0,58 & 0,10 & 1,55 & 1,17 & 0,03 & 0,24 & 0,011 & 0,11 \\
\hline & & & & & & & & & & \\
Jaulges-Villiers- & & & & & & & & & \\
Vineux n=3 & 69,15 & 21,15 & 5,55 & 1,26 & 0,31 & 0,60 & 0,17 & 1,65 & 0,013 & 0,13 \\
Ecarts-types & 1,93 & 1,85 & 1,05 & 0,04 & 0,06 & 0,05 & 0,00 & 0,11 & 0,005 & 0,07 \\
\hline & & & & & & & & & & \\
Centre (Lezoux?) & & & & & & & & & & \\
n=2 & 57,63 & 19,67 & 6,18 & 0,83 & 9,25 & 1,26 & 0,31 & 3,42 & 0,08 & 0,45 \\
Ecarts-types & 1,45 & 0,35 & 0,05 & 0,02 & 0,30 & 0,00 & 0,03 & 0,28 & 0,025 & 0,30 \\
\hline
\end{tabular}

